\title{
Reduced mucosal side-effects of acetylsalicylic acid after conjugation with tris-hydroxymethyl-aminomethane. Synthesis and biological evaluation of a new anti-inflammatory compound
}

\author{
Gabriella Varga ${ }^{a}$, Norbert Lajkó ${ }^{\mathrm{a}}$, Melinda Ugocsai ${ }^{\mathrm{a}}$, Dániel Érces ${ }^{\mathrm{a}}$, Gyöngyi Horváth ${ }^{\mathrm{b}}$, \\ Gábor Tóth ${ }^{\mathrm{c}}$, Mihály Boros ${ }^{\mathrm{a}, *}$, Miklós Ghyczy ${ }^{\mathrm{d}, 1}$ \\ ${ }^{a}$ Institute of Surgical Research, Faculty of Medicine, University of Szeged, H-6720, Szeged, Szókefalvi-Nagy Béla u. 6, Hungary \\ b Department of Physiology, Faculty of Medicine, University of Szeged, H-6720, Szeged, Dóm tér 10, Hungary \\ ${ }^{c}$ Department of Medical Chemistry, Faculty of Medicine, University of Szeged, H-6720, Szeged, Dóm tér 8, Hungary \\ d Pax Forschung GmbH, Im Rapsfeld 23, 50933 Cologne, Germany
}

\section{A R T I C L E I N F O}

\section{Article history:}

Received 26 February 2016

Received in revised form

4 April 2016

Accepted 11 April 2016

Keywords:

Aspirin

Gastritis

Inflammation

Microcirculation

Cytokines

Rat

\begin{abstract}
A B S T R A C T
Acetylsalicylic acid (ASA) causes adverse haemorrhagic reactions in the upper gastrointestinal (GI) tract, and previous results have suggested that combination therapy with 2-amino-2-(hydroxymethyl)-1,3propanediol (Tris) could provide protection in this scenario. Based on this hypothesis, our aim was to develop a new compound from ASA and Tris precursors and to characterize the biological effects of ASATris and the derivatives ASA-bis- and mono-hydroxymethyl-aminomethane (ASA-Bis, ASA-Mono, respectively) using in vivo and in vitro test systems.

ASA or ASA conjugates $(0.55 \mathrm{mmol} / \mathrm{kg}$, each) were administered intragastrically to Sprague-Dawley rats. Changes in the mucosal structure and in the serosal microcirculation were detected by in vivo imaging techniques, the plasma TNF-alpha, tissue xanthine oxidoreductase and myeloperoxidase activities, and liver cytochrome $c$ changes were also determined. In two separate series, platelet aggregation and carrageenan arthritis-induced inflammatory pain were measured in control, ASA and ASA-Tristreated groups.

Severe mucosal injury and a significant decrease in serosal red blood cell velocity developed in the ASA-treated group and an $\sim 2$-fold elevation in proinflammatory mediator levels evolved. ASA-Tris did not cause bleeding, microcirculatory dysfunction, mucosal injury or an elevation in proinflammatory markers. The ASA-Mono and ASA-Bis conjugates did not cause macroscopic bleeding, but the inflammatory activation was apparent. ASA-Tris did not influence the cyclooxygenase-induced platelet aggregation significantly, but the inflammatory pain was reduced as effectively as in the case of equimolar ASA doses.

ASA-Tris conjugation is an effective approach through which the GI side-effects of ASA are controlled by decreasing the cytokine-mediated progression of pro-inflammatory events.
\end{abstract}

(c) 2016 Elsevier B.V. All rights reserved.

\footnotetext{
* Corresponding author.

E-mail addresses: varga.gabriella.1@med.u-szeged.hu (G. Varga), lajkonorbert91@gmail.com (N. Lajkó), lindaugocsai@gmail.com (M. Ugocsai), erces.daniel@med.u-szeged.hu (D. Érces).

horvath.gyongyi@med.u-szeged.hu (G. Horváth),

toth.gabor@med.u-szeged.hu (G. Tóth), boros.mihaly@med.u-szeged.hu (M. Boros) ghyczy@t-online.de (M. Ghyczy).

1 Pax Forschung GmbH (Miklós Ghyczy) is the Applicant and Proprietor of European patent application EP 2889286A1 and International patent application WO 2015/101501 (PCT/EP2014/078296) entitledrmaceutically active compound for use as anti-inflammatory agent.
}

\section{Introduction}

Aspirin or acetylsalicylic acid (ASA) is a prototype of non-steroidal anti-inflammatory drugs (NSAIDs), and one of the most widely used medications in general medical practice. Higher doses are analgesic, antipyretic and anti-inflammatory, while lower doses are employed for the prevention of cardiovascular thrombotic events (Baigent et al., 2009). ASA is potent inhibitor of the constitutive isoform of the platelet enzyme cyclooxygenase 1 (COX-1), but it presents a variety of other pharmacological activities, such as the reduced synthesis of coagulation factors (Meade et al., 1992; Schrör et al., 1997).

Unfortunately, the side-effects of such an efficient compound 

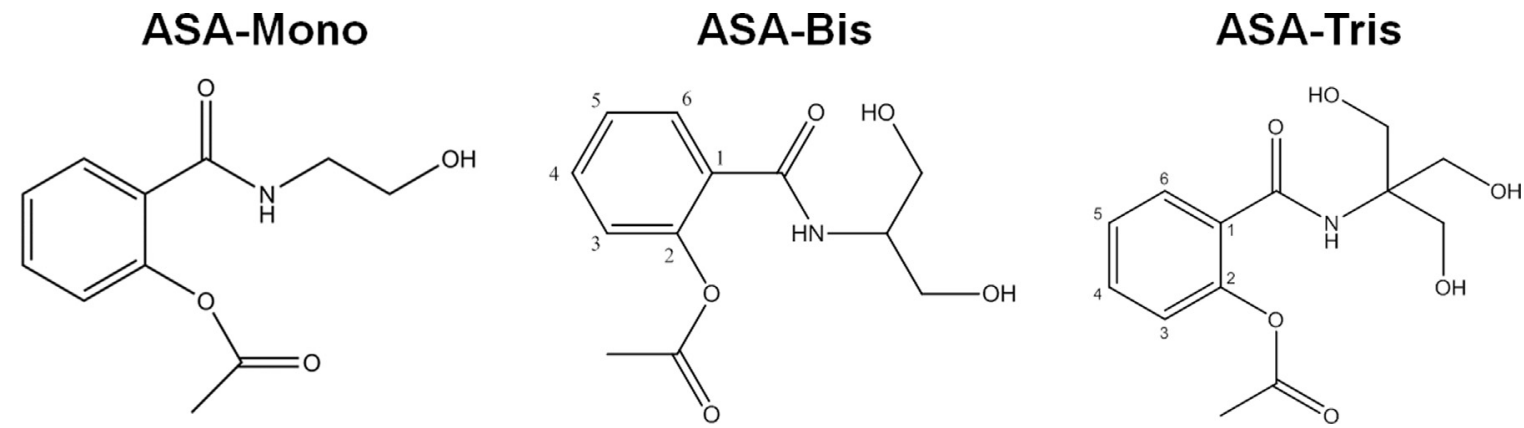

Fig. 1. Structural formulae of the developed ASA conjugates.

are also significant. Most importantly, regular ASA usage can lead to upper gastrointestinal (GI) tract toxicity (Sørensen et al., 2000, Delaney et al., 2007). The adverse effects include mild to severe functional and structural damage of the mucosal layers (Appleyard et al., 2002), which can result in haemorrhage, the development of ulcers or even perforation. The causes of bleeding are usually multiple, but two distinct mechanisms have been revealed with some degree of certainty, an ASA-induced irreversible inhibition of COX-1, and a non-prostaglandin-mediated, direct topical irritation causing alterations in the endothelial function and mucosal permeability (Hochain et al., 2000). Moreover, ASA can influence the mitochondrial respiratory activity and thiol redox functions, leading to apoptosis-inducing signals and changes in mitochondrial membrane potential (Nulton-Persson et al., 2004; Raza et al., 2012; Redlak et al., 2005).

Many strategies for the prevention of NSAID-related ulcerative complications have been developed to date, such as the maintenance of ASA in the ionic form or preventing it from dissolving until it reaches the small intestine, but the efficacy of these approaches is still not optimum. Hence, it is accepted that new, effective formulations with reduced side-effects would be of clinical interest and therapeutic importance.

The amino-alcohol tris-hydroxymethyl-aminomethane (Tris), 2-amino-2-(hydroxymethyl)-1,3-propanediol) has well-known applications in biochemistry (Gomori et al., 1955). Tris is commonly used as a component of buffer solutions effective between pH 7.07 and 9.07. The Tris buffer-mediated effects are associated with significant influences on an arterial $\mathrm{pH}$ and base deficit and a decrease in the partial tension of arterial carbon dioxide, a preferred alternative in patients with mixed acidosis (Hoste et al., 2005; Kallet et al., 2000). Besides an influence on acidaemia, Tris may inhibit enzyme activities, such as aminopeptidases and alphaamylases (Desmarais et al., 2002; Ghalanbor et al., 2008).

In view of this background, our previous data and an additional literature review, it seemed plausible that a new drug synthesized from ASA and Tris precursors might favourably affect the degree of ASA-induced mucosal damage. The primary objective of the present study was to develop an agent that exhibits bioactivity against inflammation, but is devoid of haemorrhagic side-effects in the upper GI tract. We also synthesized and tested compounds derived from ASA and mono- and bis-hydroxymethyl-aminomethane precursors to acquire comparative information and possible clues as to the mode of action. Additionally, we performed a cross-sectional evaluation study on the analgesic and antithrombotic effectiveness of ASA-Tris as compared with ASA in the rat.

\section{Materials and methods}

\subsection{The chemical synthesis of ASA-Mono, ASA-Bis and ASA-Tris conjugates}

The synthesis of the compounds and the supporting ${ }^{1} \mathrm{H}$ NMR and HPLC studies were carried out in the Department of Medical Chemistry, University of Szeged. Details of the synthetic procedures and structural characterizations are described in the Supplementary file 1 .

Briefly, ASA was dissolved in absolute tetrahydrofuran and cooled to $-15{ }^{\circ} \mathrm{C}$. Isobutyl chloroformate and triethylamine were added under stirring. After stirring at $-15^{\circ} \mathrm{C}$ for $25 \mathrm{~min}$, an equimolar amount of ethanolamine, or 2-amino-1,3-propanediol or tris-hydroxymethyl-aminomethane was added and the mixture was stirred for an additional $1 \mathrm{~h}$ at $0{ }^{\circ} \mathrm{C}$, and then at room temperature overnight. The reaction mixtures were filtered, evaporated and the resulting crystalline materials were washed with diethyl ether-hexane, resulting in the pure products (Fig. 1).

\subsection{In vivo studies}

The experiments were performed in three different series on a total of 75 male Sprague-Dawley rats (average weight $200 \mathrm{~g} \pm 10 \mathrm{~g}$ ) housed in plastic cages in a thermoneutral environment $\left(21 \pm 2{ }^{\circ} \mathrm{C}\right)$ under a 12 -h dark-light cycle. The animals were kept on normal laboratory chow and then fed with a carbohydrate-rich diet (bread rolls) for 3 days prior to the experiments.

The experimental protocols were in accordance with EU directive 2010/63 for the protection of animals used for scientific purposes and were approved by the National Scientific Ethical Committee on Animal Experimentation (National Competent Authority) with the licence number V./146/2013. This study also complied with the criteria of the US National Institutes of Health Guidelines for the Care and Use of Laboratory Animals.

\subsubsection{Experimental protocol 1}

35 animals were randomly allocated into 5 groups ( $n=7$, each). Group 1 served as vehicle-treated control ( $10 \mathrm{ml} / \mathrm{kg}$ buffered $0.11 \mathrm{M}$ potassium hydroxide $(\mathrm{KOH})$ was given orally three times daily on three consecutive days). In group 2, high doses of ASA solution $(0.55 \mathrm{mmol} / \mathrm{kg}$, in a volume of $10 \mathrm{ml} / \mathrm{kg}$; three times daily for 3 days) were gavaged via a flexible oesophageal tube to the animals. After the treatments, the animals were always returned to their cages and were fed ad libitum with a carbohydrate-rich diet.

Groups 3-5 were treated with the ASA-conjugates in equimolar doses to ASA $(0.55 \mathrm{mmol} / \mathrm{kg}$, in a volume of $10 \mathrm{ml} / \mathrm{kg}$; three times daily for 3 days), group 3 was treated with ASA-Mono; group 4 with ASA-Bis and group 5 with ASA-Tris.

On day $3,2 \mathrm{~h}$ after the last treatments, the animals were anaesthetized with sodium pentobarbital $(50 \mathrm{mg} / \mathrm{kg}$ i.p.). For instrumentation, the animals were placed in a supine position on heating pads, and the trachea and right jugular vein were cannulated to secure spontaneous breathing and i.v. administration of fluids and fluorescence dye, respectively. After a midline abdominal incision, intravital videomicroscopy was performed to examine the microcirculatory changes in the gastric serosa. The 
stomach was then incised along a greater curvature and rinsed with saline to remove the gastric contents. In each group, in vivo histology of the gastric mucosa was performed by confocal laser scanning endomicroscopy (CLSEM). At the end of the protocol, tissue biopsies were obtained from the stomach and the liver, and blood samples $(0.5 \mathrm{ml})$ were taken from the inferior caval vein.

\subsubsection{Direct measurements on the gastric microcirculation}

The orthogonal polarization spectral (OPS) imaging technique (Cytoscan A/R, Cytometrics, Philadelphia, PA, USA) was used for noninvasive visualization of the serosal microcirculation of the stomach. This technique utilizes reflected polarized light at the wavelength of the isobestic point of oxy- and deoxyhaemoglobin $(548 \mathrm{~nm})$. As polarization is preserved in reflection, only photons scattered from a depth of $2-300 \mu \mathrm{m}$ contribute to image formation. A 10x objective was placed onto the serosal surface of the stomach, and microscopic images were recorded with an S-VHS video recorder 1 (Panasonic AG-TL 700; Matsushita Electric Ind. Co. Ltd, Osaka, Japan). Quantitative assessment of the microcirculatory parameters was performed off-line by frame-to-frame analysis of the videotaped images. Red blood cell velocity (RBCV; $\mu \mathrm{m} / \mathrm{s}$ ) changes in the postcapillary venules were determined in three separate fields by means of a computer-assisted image analysis system (IVM Pictron, Budapest, Hungary).

\subsubsection{In vivo detection of mucosal damage}

The extent of damage of the gastric mucosa was evaluated by means of fluorescence CLSEM (Five1, Optiscan Pty. Ltd., Melbourne, Victoria, Australia) developed for in vivo histology. The analysis was performed twice, separately by two investigators (GV and $\mathrm{NL}$ ). The mucosal surface of the stomach was surgically exposed and laid flat for examination. The microvascular structure was recorded after the iv administration of $0.3 \mathrm{ml}$ of fluorescein isothiocyanate-dextran (FITC-dextran, $150 \mathrm{KDa}, 20 \mathrm{mg} / \mathrm{ml}$ solution dissolved in saline, Sigma Chem.). The objective of the device was placed onto the mucosal surface of the stomach and confocal imaging was performed $5 \mathrm{~min}$ after dye administration (1 scan/ image, $1024 \times 512$ pixels and $475 \times 475 \mu \mathrm{m}$ per image). The changes in the mucosal architecture were examined following topical application of the fluorescent dye acridine orange (Sigma-Aldrich Inc, St. Louis, MO, USA). The surplus dye was washed off the mucosal surface of the stomach with saline 2 min before imaging.

Non-overlapping fields gastric mucosa were processed in ASAtreated animals and compared with the samples of the control or ASA conjugate-treated groups by using a semiquantitative scoring system as described previously (Kovács et al., 2012). We employed three criteria: I. the structure of the microvessels $(0=$ normal, $1=$ dye extravasation, but the vessel structure recognizable, 2 =destruction, and the vessel structure unrecognizable); II. oedema $(0=$ no oedema, $1=$ moderate epithelial swelling, $2=$ severe oedema); and III. epithelial cell outlines $(0=$ normal, clearly, welldefined outlines, 1 =blurred outlines, 2 =lack of normal cellular contours).

\subsubsection{Experimental protocol 2. Platelet aggregation measurements}

The efficacy of ASA-Tris was tested on platelet functions in a separate series; the protocol was identical to that described above. Briefly, 15 animals were randomly allocated into 3 groups $(n=5$, each), group 1 served as the vehicle-treated control, and repeated doses of ASA, or ASA-Tris solution $(0.55 \mathrm{mmol} / \mathrm{kg}$, in a volume of $10 \mathrm{ml} / \mathrm{kg}$; three times daily for 3 days) were gavaged via a flexible oesophageal tube to the animals in groups 2 and 3, respectively. Two $\mathrm{h}$ after the last treatment, the animals were anaesthetized with sodium pentobarbital and blood samples were taken from the inferior caval vein. The measurement of platelet aggregation was carried out with multiplate electrode aggregometry
(Multiplate analyzer, Roche, Basel, Switzerland). Briefly, after platelet activation the analyzer records the impedance between two electrodes reflecting thrombocyte aggregation on the surface of the electrodes. $300 \mu \mathrm{l}$ blood samples were placed in hirudinized tubes to perform multiplate aggregation tests using arachidonic acid, the substrate of COX, which subsequently forms the platelet activator thromboxane $A_{2}$ (ASPI-test), collagen, which leads to a release of endogenous arachidonic acid and $\mathrm{TXA}_{2}$ via the collagen receptors (Col-test), and the adenosine-diphosphate (ADP)-induced platelet activation test (ADP-test).

\subsubsection{Experimental protocol 3}

The analgesic effect of ASA-Tris was tested on the carrageenaninduced paw inflammation model, using 18 male SPRD rats randomly allocated into 3 groups $(n=6$, each). Group 1 served as positive control, group 2 was treated with ASA, and group 3 with ASA-Tris conjugate in doses identical to those described above. In groups 2 and 3 , the treatment was administered $3 \mathrm{~h}$ after the induction of paw inflammation by the injection of carrageenan $(300 \mu \mathrm{g} / 30 \mu \mathrm{l})$ into the tibiotarsal joint of the right hind limb. All treatments were given to gently restrained conscious animals via a 27-gauge needle without anaesthesia.

The analgesic effects of ASA and the ASA-Tris derivative treatments were determined by using a dynamic plantar aesthesiometer (mod-37450; Ugo Basile, Comerio, Italy). Before baseline measurements, each rat was habituated to a testing box with a wiremesh grid floor for at least $20 \mathrm{~min}$. The measurements were performed with a straight metal filament (diameter $0.5 \mathrm{~mm}$ ) that exerts an increasing upward force at a constant rate $(4.25 \mathrm{~g} / \mathrm{s})$ with a maximum cut-off force of $50 \mathrm{~g}$. The filament was placed under the plantar surface of the right hind paw. Measurements were stopped when the paw was withdrawn, and results were expressed as paw withdrawal thresholds in grams.

The baseline measurements were performed 15 min before the induction of inflammation, while the development of inflammation was investigated $3 \mathrm{~h}$ after the carrageenan induction. The ASA or ASA-Tris treatments were administered $10 \mathrm{~min}$ after the second measurements; subsequent measurements were performed at 60 , 120 and $180 \mathrm{~min}$.

\subsection{In vitro studies}

\subsubsection{Preparation of tissue biopsies}

Gastric biopsies kept on ice were homogenized in phosphate buffer ( $\mathrm{pH}$ 7.4) containing $50 \mathrm{mM}$ Tris- $\mathrm{HCl}$ (Reanal, Budapest, Hungary), $0.1 \mathrm{mM}$ EDTA, $0.5 \mathrm{mM}$ dithiotreitol, $1 \mathrm{mM}$ phenylmethylsulfonyl fluoride, $10 \mu \mathrm{g} / \mathrm{ml}$ soybean trypsin inhibitor and $10 \mu \mathrm{g} / \mathrm{ml}$ leupeptin (Sigma-Aldrich $\mathrm{GmbH}$, Germany). The homogenate was centrifuged at $4{ }^{\circ} \mathrm{C}$ for $20 \mathrm{~min}$ at $24,000 \mathrm{~g}$ and the supernatant was loaded into centrifugal concentrator tubes (Amicon Centricon-100; 100,000 MW cut-off ultrafilter). The activity of xanthine oxidoreductase (XOR) was determined in the ultrafiltered supernatant, while that of myeloperoxidase (MPO) was measured on the pellet of the homogenate.

\subsubsection{Tissue MPO activity}

The activity of MPO as a marker of tissue leukocyte infiltration was measured on the pellet of the homogenate (Kuebler et al., $1996)$. Briefly, the pellet was resuspended in $\mathrm{K}_{3} \mathrm{PO}_{4}$ buffer $(0.05 \mathrm{M}$; $\mathrm{pH}$ 6.0) containing $0.5 \%$ hexa-1,6-bis-decyltriethylammonium bromide. After three repeated freeze-thaw procedures, the material was centrifuged at $4{ }^{\circ} \mathrm{C}$ for $20 \mathrm{~min}$ at $24,000 \mathrm{~g}$ and the supernatant was used for MPO determination. Subsequently, $0.15 \mathrm{ml}$ of 3,3',5,5'-tetramethylbenzidine (dissolved in DMSO; $1.6 \mathrm{mM}$ ) and $0.75 \mathrm{ml}$ of hydrogen peroxide (dissolved in $\mathrm{K}_{3} \mathrm{PO}_{4}$ buffer; $0.6 \mathrm{mM}$ ) were added to $0.1 \mathrm{ml}$ of the sample. The reaction led to the 
hydrogen peroxide-dependent oxidation of tetramethylbenzidine, which could be detected spectrophotometrically at $450 \mathrm{~nm}$ (UV1601 spectrophotometer; Shimadzu, Kyoto, Japan). MPO activities were measured at $37^{\circ} \mathrm{C}$; the reaction was stopped after 5 min by the addition of $0.2 \mathrm{ml}$ of $\mathrm{H}_{2} \mathrm{SO}_{4}(2 \mathrm{M})$ and the resulting data were referred to the protein content.

\subsubsection{XOR activity}

The XOR activity was determined in the ultrafiltered, concentrated supernatant by a fluorometric kinetic assay based on the conversion of pterine to isoxanthopterine in the presence (total XOR) or absence (xanthine oxidase activity) of the electron acceptor methylene blue (Beckman et al., 1989).

2.3.3.1. Measurement of tissue nitric oxide (NO) products. Nitrite and nitrate $\left(\mathrm{NO}_{\mathrm{x}}\right)$, stable end-products of $\mathrm{NO}$, were determined in the gastric homogenate by the Griess reaction. This assay depends on the enzymatic reduction of nitrate to nitrite, which is then converted into a coloured azo compound that is detected spectrophotometrically at $540 \mathrm{~nm}$. Total $\mathrm{NO}_{\mathrm{x}}$ was calculated and expressed as $\mu \mathrm{mol} /(\mathrm{mg}$ protein) (Moshage et al., 1995).

\subsubsection{Measurement of malondialdehyde (MDA) level}

The degree of lipid peroxidation was estimated via the amount of MDA, a marker of oxidative damage of lipid membranes. MDA level was measured through the reaction with thiobarbituric acid and the values were corrected for the tissue protein content. The MDA concentration was determined on a standard curve (nmol/ ml) (Placer et al., 1966).

\subsubsection{Measurement of cytochrome c oxidase release}

Cytochrome c oxidase release was calculated via the time-dependent oxidation of cytochrome $\mathrm{c}$ at $550 \mathrm{~nm}$ as described previously (Szarka et al., 2004). Briefly, liver and gastric tissue samples were homogenized in 10x ice-cold MitOx2 medium with a Potter grinder, and then centrifuged at $800 \mathrm{~g}$ for $5 \mathrm{~min}$ at $4{ }^{\circ} \mathrm{C}$. $50 \mu$ supernatant was added to $2.5 \mathrm{ml}$ cytochrome c stock solution (10.6 mg cytochrome c dissolved in $20 \mathrm{ml}$ distilled water) (Sigma-Aldrich, Budapest, Hungary) and the decrease in optical density at $550 \mathrm{~nm}$ was measured spectrophotometrically during 1 -min intervals at 0,30 and $60 \mathrm{~min}$.

\subsubsection{Plasma TNF- $\alpha$ level measurements}

Blood samples $(0.5 \mathrm{ml})$ were taken from the inferior caval vein into precooled, heparizined $(100 \mathrm{U} / \mathrm{ml})$ polypropylene tubes, centrifuged at $1000 \mathrm{~g}$ at $4{ }^{\circ} \mathrm{C}$ for $30 \mathrm{~min}$ and then stored at $-70{ }^{\circ} \mathrm{C}$ until assay. Plasma TNF-alpha concentrations were determined in duplicate by means of a commercially available enzyme-linked immunosorbent assay (Quantikine ultrasensitive ELISA kit for rat TNF-alpha; Biomedica Hungaria Kft, Budapest, Hungary). The minimum detectable level was less than $5 \mathrm{pg} / \mathrm{ml}$, and the interassay and intra-assay coefficients of variation were less than $10 \%$.

\subsection{Statistical analysis}

Data analysis was performed with a statistical software package (SigmaStat for Windows, Jandel Scientific, Erkrath, Germany). Friedman repeated measures analysis of variance on ranks was applied within groups. Time-dependent differences from the baseline for each group were assessed by Dunn's method. Differences between groups were analysed with Kruskal-Wallis oneway analysis of variance on ranks, followed by Dunn's method for pairwise multiple comparison. In the Figures, median values and 75th and 25th percentiles are given; $\mathrm{P}$ values $<0.05$ were considered significant.

\section{Results}

\subsection{Estimation of the severity of gastritis}

ASA treatment resulted in manifest, visible bleeding and significant loss of bodyweight relative to the control group. The ASAMono, ASA-Bis and ASA-Tris treatments did not cause significant changes in bodyweight (Table 1 ).

\subsection{In vivo detection of gastric mucosal injury}

The gastric microvessels were visualized by FITC-dextran administration, while the morphology of the gastric mucosa was examined by the topical application of acriflavine dye (Fig. 2). In the control group, the network of capillaries and gastric mucosal epithelium exhibited a normal pattern $(\mathrm{M}=0 ; \mathrm{p} 25=0 ; \mathrm{p} 75=0.15)$. The evaluation of the confocal microscopic records demonstrated significant tissue damage in the ASA-treated group in contrast with the control group (Fig. 3). The capillary network was severely injured, the fluorescent dye leakage pointed to an elevated endothelial permeability and oedema formation $(\mathrm{M}=5$; $\mathrm{p} 25=3.75$; $\mathrm{p} 75=5.25$; Figs. 2, 3). In the ASA-Mono $(\mathrm{M}=2.5 ; \mathrm{p} 25=1.8$; $\mathrm{p} 75=3)$ and ASA-Bis $(\mathrm{M}=2.5 ; \mathrm{p} 25=2 ; \mathrm{p} 75=3)$ treated groups, increased vascular permeability was observed, but this change was accompanied by a normal mucosal pattern. The ASA-Tris treatment did not lead to structural damage or morphological changes in the gastric mucosa. The loss of epithelium accompanying ASA administration was not present (Figs. 2,3), the changes were similar to those observed in the untreated control group $(M=0$; $\mathrm{p} 25=0 ; \mathrm{p} 75=0.15$ ).

\subsection{In vivo detection of the microcirculation}

The RBCV of the serosa was measured as a quantitative marker of the gastric microcirculatory condition. The RBCV was significantly decreased in the ASA-treated group as compared with the control group. ASA-Mono and ASA-Bis treatments caused significant, but moderate reductions in the microcirculation in contrast with the control group, and these changes were significantly lower relative to that in the ASA-treated group. The ASATris treatment prevented the reduction in RBCV (Table 1).

Table 1

The effects of ASA and ASA-conjugates treatment on changes of body weight $[\mathrm{g}]$ red blood cell velocity $[\mu \mathrm{m} / \mathrm{s}]$ and TNF-alpha $[\mathrm{pg} / \mathrm{ml}]$.

\begin{tabular}{|c|c|c|c|c|}
\hline & Parameters & Body weight & $\begin{array}{l}\text { Reed blood cell } \\
\text { velocity }\end{array}$ & $\begin{array}{l}\text { TNF- } \\
\text { alpha }\end{array}$ \\
\hline \multirow[t]{2}{*}{ Control } & Median & 20 & 963 & 3.08 \\
\hline & $25 p ; 75 p$ & $12.5 ; 22.5$ & $842 ; 1042$ & $2.51 ; 3.09$ \\
\hline \multirow[t]{2}{*}{ ASA } & Median & -15 & $269^{a}$ & $6.28^{\mathrm{a}}$ \\
\hline & $25 p ; 75 p$ & $-25 ;-7.5$ & $224 ; 426$ & $4.72 ; 8.57$ \\
\hline \multirow[t]{2}{*}{ ASA-Mono } & Median & $15^{\mathrm{b}}$ & $539, a, b$ & $4.2^{a}$ \\
\hline & $25 p ; 75 p$ & $12.5 ; 20$ & 502; 657 & $3.35 ; 7.82$ \\
\hline \multirow[t]{2}{*}{ ASA-Bis } & Median & $15^{b}$ & 432 ,a,b & $1.466^{, a, b}$ \\
\hline & $25 p ; 75 p$ & 12,$5 ; 22.5$ & $406 ; 605$ & $0.59 ; 2.61$ \\
\hline \multirow[t]{2}{*}{ ASA-Tris } & Median & $15^{b}$ & $967^{b, c, d}$ & $1.94^{b, c}$ \\
\hline & $25 p ; 75 p$ & $10 ; 20$ & $882 ; 1006$ & $0.95 ; 2.53$ \\
\hline
\end{tabular}

${ }^{\mathrm{a}} \mathrm{P}<0.05$ between groups vs control group;

${ }^{\mathrm{b}} \mathrm{P}<0.05$ between ASA-Mono, ASA-Bis, ASA-Tris groups vs ASA group;

${ }^{c} \mathrm{P}<0.05$ between ASA-Mono group vs ASA-Tris group;

${ }^{\mathrm{d}} \mathrm{P}<0.05$ between ASA-Bis group vs ASA-Tris. 

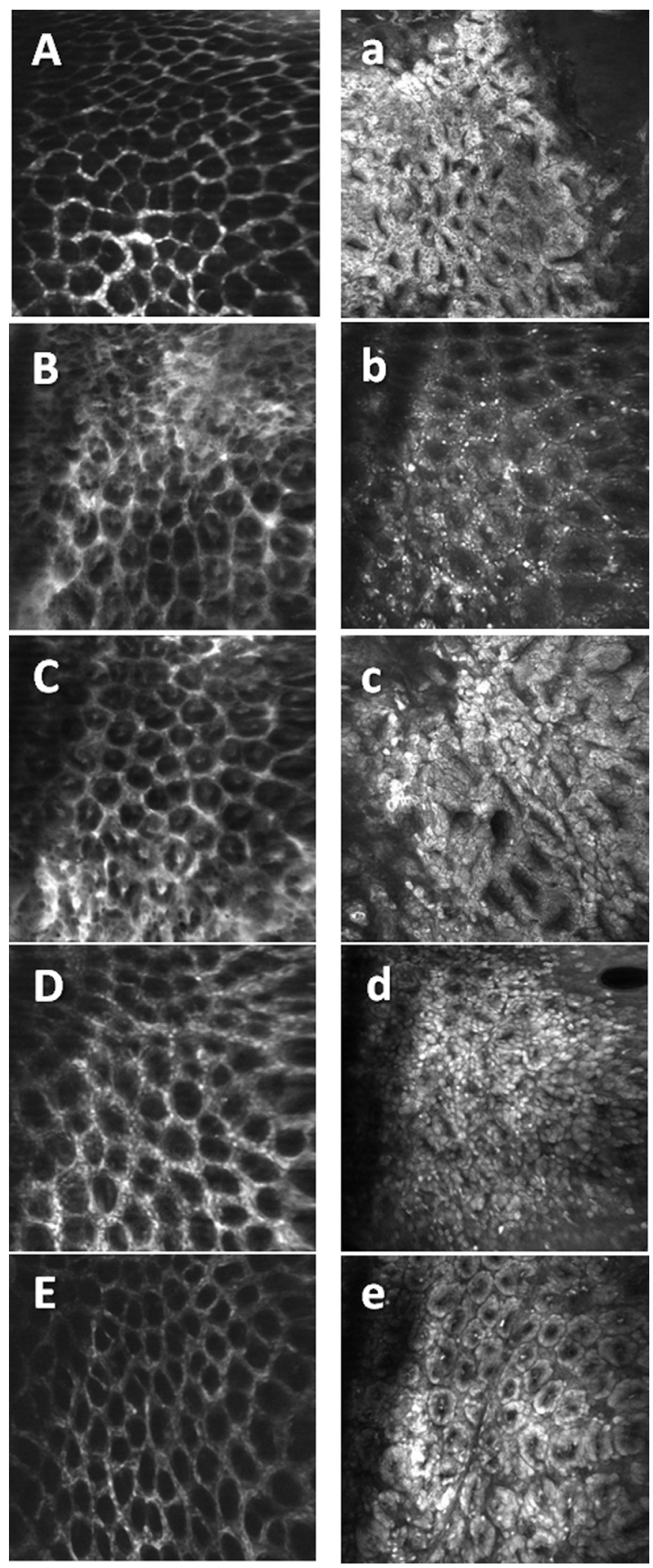

Fig. 2. Left panel In vivo histology images of the mucosal surface of the stomach recorded by confocal laser scanning endomicroscopy (CLSEM) after iv administration of FITC-dextran: A: The mucosal vasculature in the control group. B: Dye leakage from the vessel lumina after 3 days of ASA-treatment. C: 3 days of ASAMono treatment. D: 3 days of ASA-Bis treatment. E: Normal mucosal vasculature after 3 days of ASA-Tris-treatment. Right panel CLSEM after topical administration of acriflavine: a: Normal structure of the mucosa in the control group. b: Total loss of epithelium on the surface after 3 days of ASA treatment. c: Normal structure of the mucosa after 3 days of ASA-Mono treatment. d: ASA-Bis treatment. e: Normal structure of the mucosa after 3 days of ASA-TRIS treatment.

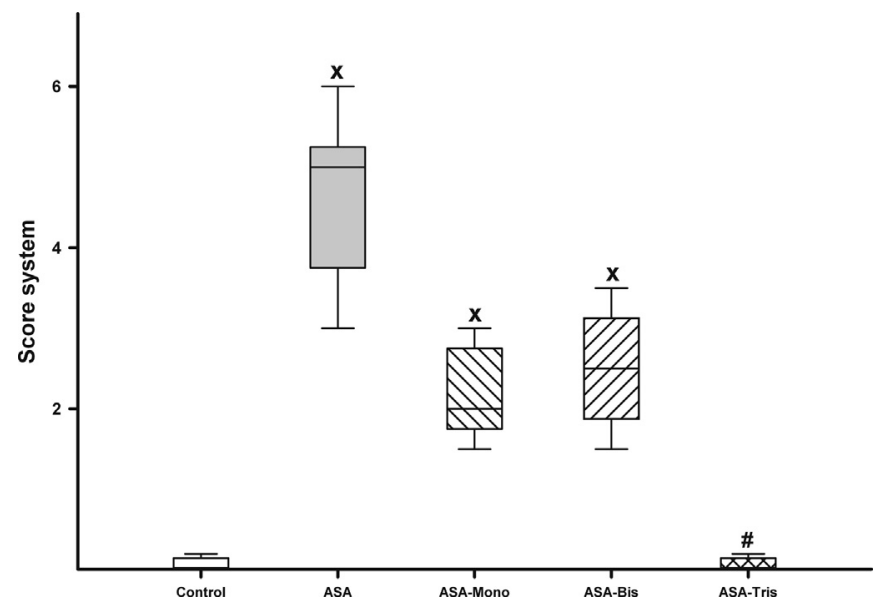

Fig. 3. Grading of in vivo histology. Control (white empty box), ASA-treated (grey empty box), ASA-Mono-treated (striped white box on the right side), ASA-Bistreated (striped white box on the left side) and ASA-Tris-treated (checked white box) groups. The plots demonstrate the median (horizontal line in the box) and the 25th (lower whisker) and 75th (upper whisker) percentiles. ${ }^{\mathrm{x}} \mathrm{P}<0.05$ between groups vs control group, ${ }^{\#} \mathrm{P}<0.05$ between ASA-treated $v s$ ASA-Tris-treated groups.

\subsection{Inflammatory enzyme activities and $\mathrm{NO}_{x}$ level}

The ASA treatment caused tissue leukocyte accumulation as revealed via measurement of the MPO activity. The median MPO activity in the control animals at the end of the observation period was 1035 ( $\mathrm{p} 25=1020 ; \mathrm{p} 75=1508) \mathrm{mU} /(\mathrm{mg}$ protein $) .2 \mathrm{~h}$ after the last ASA treatment, the gastric MPO activity was increased significantly $(\mathrm{M}=2069 ; \mathrm{p} 25=1951 ; \mathrm{p} 75=2247 \mathrm{mU} /(\mathrm{mg}$ protein $))$ relative to the control group. The ASA-Mono treatment resulted in a significant elevation in the MPO activity as compared with the control group, while the ASA-Bis and ASA-Tris $(\mathrm{M}=970$; $\mathrm{p} 25=927 ; \mathrm{p} 75=1176 \mathrm{mU} /(\mathrm{mg}$ protein $))$ treatments prevented the increase of MPO activity in the gastric tissue (Fig. 4A).

The XOR enzyme activity $2 \mathrm{~h}$ after the last ASA treatment was significantly elevated in contrast with the control group. The ASAMono treatment did not influence while the ASA-Bis and ASA-Tris treatments significantly decreased the activity of XOR (Fig. 4B).

The ASA treatment significantly elevated the $\mathrm{NO}_{\mathrm{x}}$ level in the gastric tissue relative to the control group. The elevation of $\mathrm{NO}_{\mathrm{x}}$ was significantly higher in comparison with the control group in the ASA-Mono and ASA-Bis-treated groups. ASA-Tris treatment decreased the $\mathrm{NO}_{\mathrm{x}}$ elevation in contrast with the non-treated or other ASA conjugate-treated groups (Fig. 4C).

\subsection{MDA and cytochrome c oxidase level}

The MDA level was significantly increased in the gastric tissue in the ASA-treated group as compared with the control group. The ASA-Mono, ASA-Bis, or ASA-Tris treatment significantly prevented the elevation in MDA level (Fig. 5A).

Cytochrome $c$ release from the mitochondria as an indicator of mitochondrial membrane damage was determined in the liver and gastric tissues. The hepatic cytochrome c level was significantly increased in the ASA- or ASA-Mono-treated groups as compared with the control group. The ASA-Bis or ASA-Tris treatment significantly prevented the elevation in cytochrome $c$ release (Fig. 5B). The cytochrome c release was also measured in the gastric tissue (Fig. 5C). In these samples, the cytochrome c level was significantly elevated as a result of ASA treatment, while the release of cytochrome c could not be demonstrated in the ASA-Tris group. 

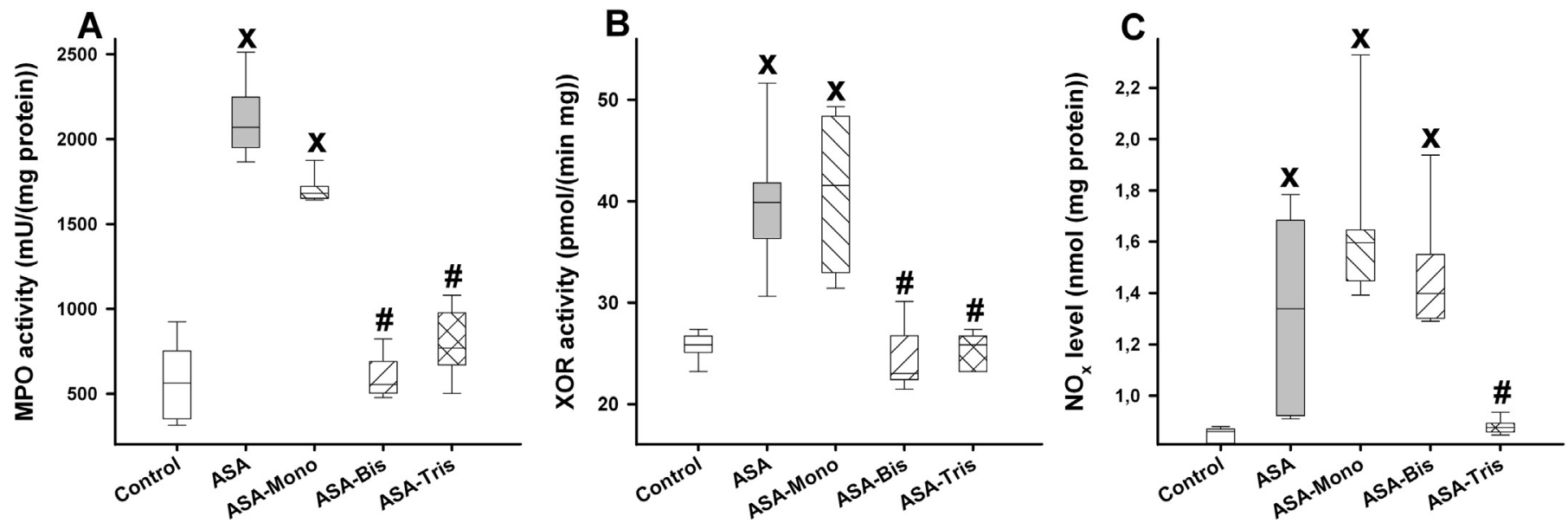

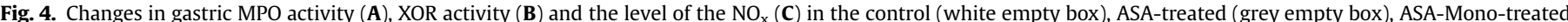

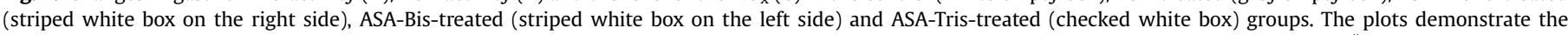

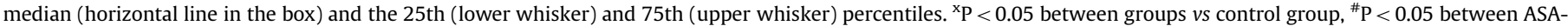
treated vs ASA-Tris-treated groups.
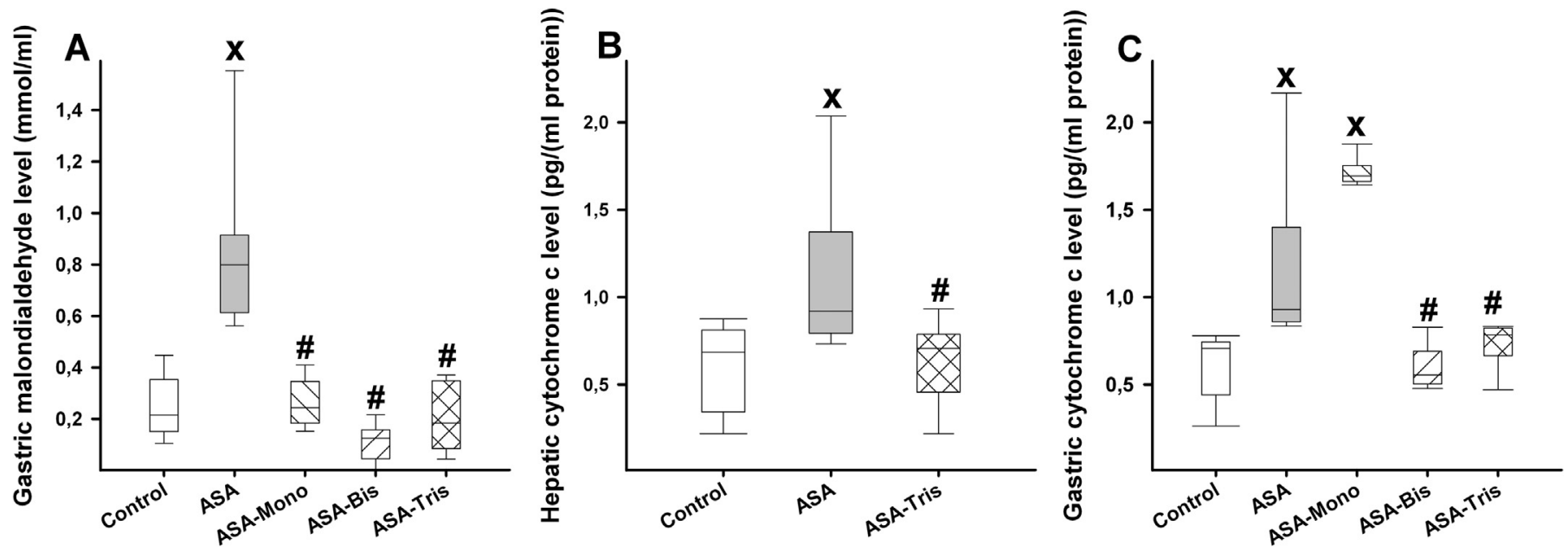

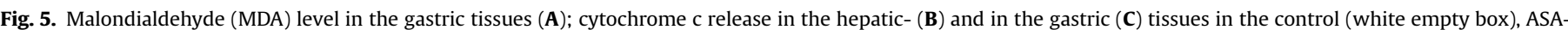

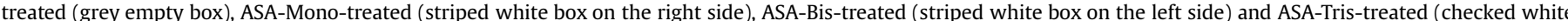

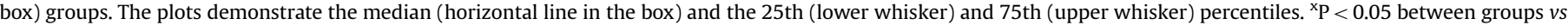
control group, ${ }^{\#} \mathrm{P}<0.05$ between ASA-treated vs ASA-Tris-treated groups.

\subsection{Changes in plasma TNF- $\alpha$ level}

The plasma level of TNF- $\alpha$ was significantly increased after ASA and ASA-Mono administration as compared with the control group. The plasma level of TNF- $\alpha$ in the ASA-Bis-, or ASA-Tristreated groups was kept at a significantly lower level relative to the ASA-treated group (Table 1).
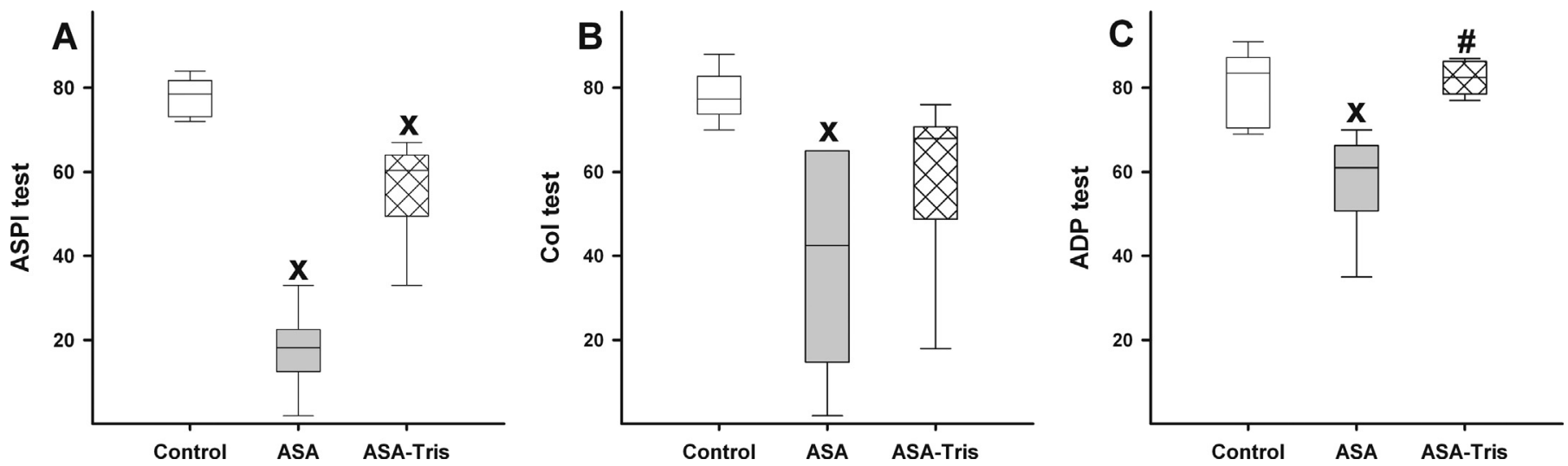

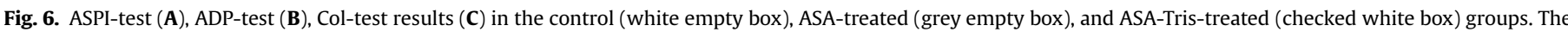

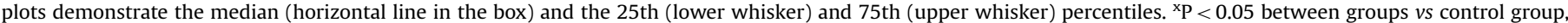
${ }^{\#} \mathrm{P}<0.05$ between ASA-treated vs ASA-Tris-treated groups. 


\subsection{Changes in platelet aggregation}

To test different pathways of platelet aggregation, the ASPI-test, ADP-test and Col-test were used, which demonstrated an approximately $80 \%$ decrease in platelet function after ASA treatment as compared with the control group. After ASA-Tris treatment, the ASPI-test showed that the rate of thrombocyte aggregation was $20 \%$ lower than in the control group, but the Col test and ADP test results were not affected (Fig. 6).

\subsection{Changes in nociception}

A significant decrease in the paw pressing force was detected in all groups $3 \mathrm{~h}$ after carrageenan administration on the treated side, referring to the development of inflammation-induced pain. Acute, single-dose ASA or ASA-Tris administrations significantly increased these values. This effect was transitional after ASA treatment, while equimolar ASA-Tris resulted in a sustained and prolonged effect lasting until the end of the 180-min observation period (Fig. 7).

\section{Discussion}

Ulcerative lesions and bleeding are major side-effects of ASA therapies in the upper GI tract. The physiological barrier mechanism of the mucosa is vital for the homeostasis of the body (Foitzik et al., 1999), and many research attempts have therefore been made to reduce the incidence of haemorrhagic gastroduodenal damage. It is well recognized that early events in the pathogenesis are microvascular leakage with leukocyte accumulation in the mucosa (Wallace et al., 1990). ASA might also alter the gastric mucosal cell functions (Alino et al., 1986; Pizzuto et al., 1997) and paradoxically stimulating the $\mathrm{Ca}^{2+}$-dependent TNF- $\alpha$ release by activated macrophages which has direct cytotoxic effects (Fiorucci et al., 1998).
Over recent decades, many advances have been made in the delivery of NSAID medications to make the treatment safer. Recent trends of drug development have included "modified release" enteric coating formulations to prevent gastric or duodenal ulceration. Co-therapy of NSAIDs with other compounds has also been used. Is noteworthy that Tris has also been tried as a cationic saltforming agent in 1:1 combination in the NSAID ketorolac tromethamine, to increase the solubility of the formulation (Mroszczak et al., 1987). The main goal of the present study was different; the intention was to develop a novel anti-inflammatory compound, with ASA-like therapeutic efficacy and reduced severity of GI tract-damaging side-effects. To this end, we synthesized a new molecule from the ASA and Tris precursors, and the data demonstrate that the ASA-Tris conjugate works well as an anti-inflammatory compound with much less damaging effect on the gastric mucosa as compared with the original ASA. It should be added that the possibility of a chemical reaction occurring between an NSAID and its Tris partner has never been investigated previously.

In this study, we characterized the ASA-induced haemorrhagic mucosal injury via in vitro assays and in vivo tests in rodents. Intragastric administration of ASA caused a disruption of the mucosal barrier, which was accompanied by deterioration of the serosal and mucosal microcirculation. The intravital histology confirmed the evolving endothelial injury and the associated, severe loss of epithelial cover. The significant role of PMN leukocytes in the deleterious effects of ASA was verified; the elevated MPO indicated that the PMNs are anchored in the inflamed gastric tissue. These potentially detrimental alterations were accompanied by an enhanced activity of XOR, a major reactive oxygen speciesproducing enzyme. The elevated tissue MDA and $\mathrm{NO}_{\mathrm{x}}$ levels and cytochrome $\mathrm{c}$ release were accompanied both by increased circulating plasma TNF- $\alpha$ concentrations and by significant cytochrome c release in the liver, demonstrating the spreading of inflammatory signals and the systemic aspect of a local insult.

ASA-Tris, however, did not cause neutrophil accumulation,

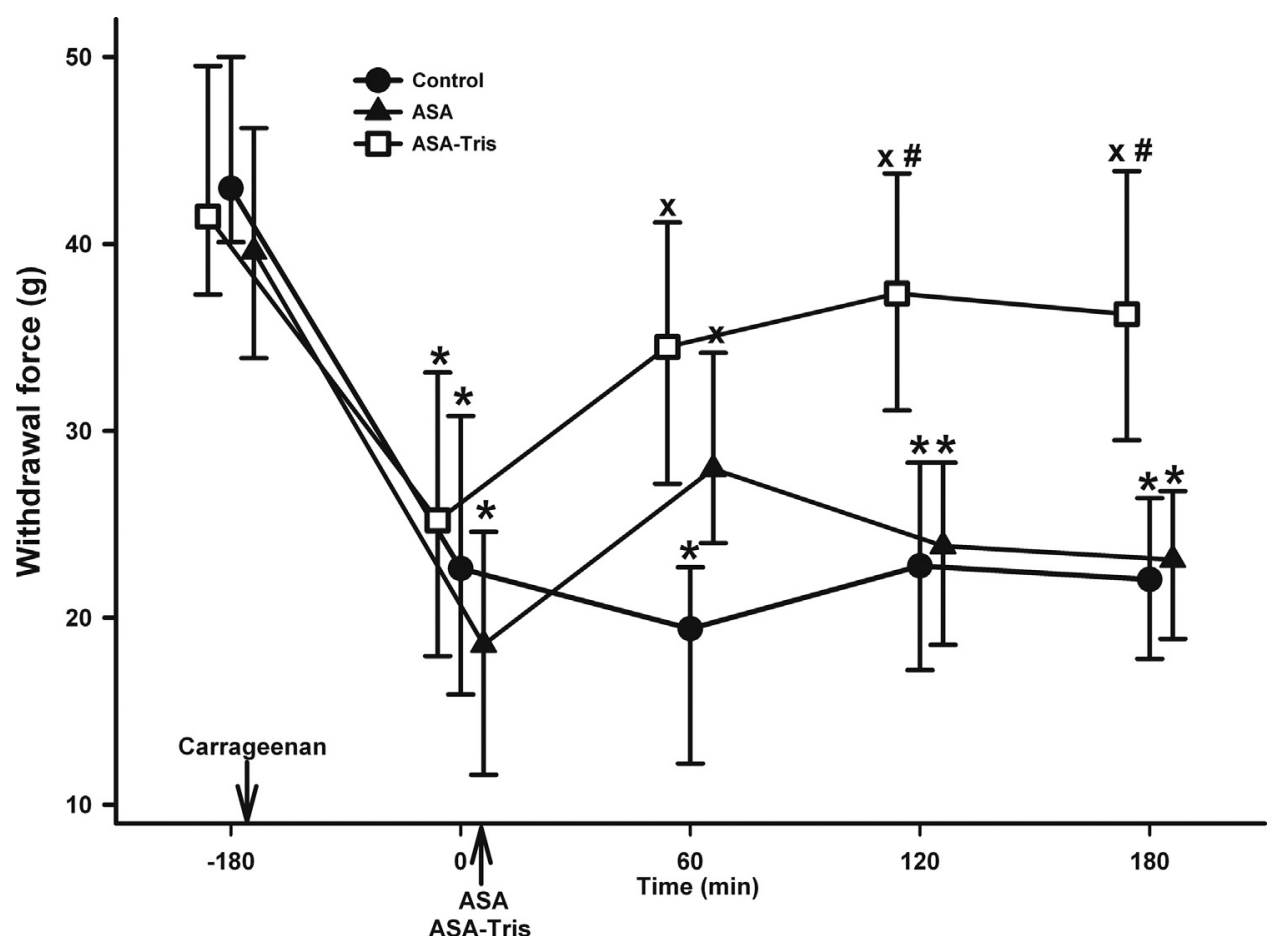

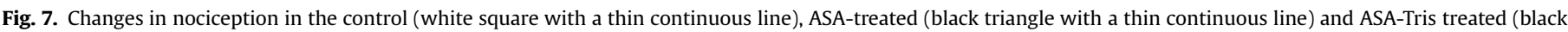

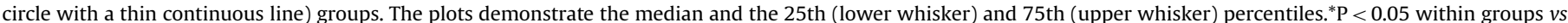
baseline values $(0 \mathrm{~h}),{ }^{\mathrm{x}} \mathrm{P}<0.05$ between groups $v s$ control group; ${ }^{\#} \mathrm{P}<0.05$ between ASA and ASA-Tris-treated groups. 
inflammatory enzyme activation or cytokine release. The ASA-Tris conjugate did not elevate the gastric MDA level, and in parallel the release of cytochrome c could not be detected in the gastric, or hepatic tissues. The in vivo histology provided visible evidence for the lack of mucosal damage after ASA-Tris administration. The ASA-Tris conjugate effectively blocked the increase of inflammatory markers and weight loss, and prevented the change in microvascular structure too.

The treatment with the ASA-Mono compound increased the MPO and XOR activity and plasma TNF- $\alpha$ levels, but the bodyweight of the animals did not change and the microcirculation was only moderately decreased. The ASA-Bis conjugate was somewhat more effective; this treatment did not induce a mucosal injury and the TNF- $\alpha$ release was lower as compared with ASA. The microvascular leakage could be repeatedly observed in the ASA-Mono and ASA-Bis groups, but not after the ASA-Tris treatments. In general, the RBCV changed in parallel with the damage of the microvessels, and thus the lowest values were detected after ASA administration; in the ASA-Mono and ASA-Bis groups, only a moderate RBCV decrease was observed, while the RBCV was kept at the control level after ASA-Tris treatment. In the ASA-Mono group, the level of MDA was not increased, but the level of cytochrome $\mathrm{c}$ was elevated. After the ASA-Bis and ASA-Tris treatments, the MDA and the cytochrome c levels were in the normal range. To give a better overview of the findings, the effects of ASA, ASA-Bis, ASA-Mono and ASA-Tris are summarized in a somewhat arbitrary, but more comprehensible numerical format (see Supplementary file 2) and these data clearly demonstrate that the reduced GI toxicity of the conjugates is in association with the specific molecular structure of the substance.

The possible anti-inflammatory and antithrombotic effects were also investigated and ASA-Tris was found to be potent in producing an anti-nociceptive effect as ASA. The platelet responses were assessed with validated and standardized methods (Boeer et al., 2010), and as expected, ASA treatment decreased the COXdependent aggregation, the collagen induced aggregation and the ADP-induced platelet activation. The ASA-Tris treatment decreased the COX-dependent aggregation to a certain degree, but it did not significantly influence the collagen-induced aggregation and the ADP-induced platelet activation. The background of the phenomenon demands further investigation, but this suggests that ASATris is most likely not a COX inhibitor, and in this case the antiinflammatory property stems from an effect different from ASA.

It is established that reactive oxygen species play an important role in the pathophysiology of acute ulceration induced by ASA (Fiorucci et al., 1998). The reactive oxygen species production can be linked to mitochondria or to other sources, such as activated leukocytes or the increased activity of cellular oxidases. The peroxidation of polyunsaturated fatty acids, an immediate reactive oxygen species-induced chain reaction, causes breakdown of the biomembranes and the loss of maintenance of the cellular steady state (De Cuyper and Joniau, 1980; Slater et al., 1984). The degree of lipid peroxidation can be estimated via the amount of MDA, a marker of oxidative damage of lipid membranes. During hypoxia or systemic inflammatory activation the progressively depressed electron transport through the inner mitochondrial membrane is accompanied by phospholipid damage and the loss of cytochrome c. Cytochrome c, attached to the inner mitochondrial membrane, becomes detached in response to a threshold disturbance in the membrane structure, which leads to activation of the apoptotic caspase cascade (Garrido et al., 2006).

The cytochrome $\mathrm{c}$ and MDA changes together are indicators of the reactive oxygen species-induced mitochondrial membrane damage, while $\mathrm{NO}_{\mathrm{x}}$ levels are indirectly linked to increased $\mathrm{NO}$ production. Collectively, the data demonstrate that oxidative and nitrosative stress reactions, which generally accompany mitochondrial membrane damage, played roles in ASA-induced mucosal injury, while ASA-Tris administration was devoid of these consequences. In other words, these data suggest that ASA-Tris did not cause significant oxidative membrane damage, or the fate of the cell towards a reactive oxygen species-producing stress condition was blocked. Our results may also refer to a maintained or preserved energetic state of the cell that can contribute to an improved cell function which finally ends in the reduction of the mucosal damage.

The mechanism of action of ASA-Tris is not known with certainty. On the one hand, the masked carboxyl group in the ASA derivatives can prevent the local irritation of the gastric mucosa. While the exact mechanism of the interaction is unknown, it seems that the effect is proportional to the alcoholic moiety, since ASA-Mono, ASA-Bis and ASA-Tris displayed an effectiveness proportional to the number of hydroxy groups in the compounds. The mechanism of action should be explored in further studies, but it has already been shown that flavonoids with multiple hydroxy groups are more effective antioxidants than those with only one. Furthermore, the operation of a redox control mechanism is also plausible where the alcoholic moiety of ASA-Tris may be the active part of a process which leads to a reduction of membrane damage. The reduced form of the tripeptide glutathione (GSH), and its ratio to the oxidized glutathione disulphide form (GSSG), is the major thiol-disulphide redox buffer system of the cell. Ethanol (or perhaps analogously, ASA-Tris), through increasing the intracellular NADH can split the disulphide GSSG leading to increased GSH, thereby increasing the low GSH/GSSG back to higher levels (Watson et al., 2011). This reaction results in a swift change from the more oxidizing redox state towards a reducing milieu, i.e. the normal condition of a healthy cell. Thirdly, the change in MDA suggests a radical-induced peroxidation reaction after ASA and direct antioxidant activity for ASA-Tris. In this case it is assumed that in this milieu the radical, if generated, abstracts a hydrogen atom from the aromatic hydroxy group of ASA-Tris rather than from a polyunsaturated fatty acid in the biomembranes of the cell.

In conclusion, we have demonstrated that a new, biologically active product with promising pharmacological properties can be formed from ASA and Tris precursors. The study has a number of limitations that may warrant discussion. For instance, the biochemical interactions between ASA-Tris and possible target molecules need to be considered in more detail, and the reactions associated with reactive oxygen species generation should be investigated further. Many other details remain missing at present, metabolism and tissue penetration data should be collected, but the available evidence demonstrates that ASA-Tris might provide therapeutic benefit and efficiency by targeting inflammatory changes without significant side-effects on the gastric mucosa.

\section{Acknowledgements}

The authors are grateful to Ms. Ágnes Fekete, Csilla Mester, Nikolett Beretka and Lilla Kovács, Zoltánné Szelepcsenyi for skilful assistance. The study was supported by the Hungarian Science Research Fund OTKA K104656. Competing interest: Pax Forschung $\mathrm{GmbH}$ (Miklos Ghyczy) is the Applicant and Proprietor of European patent application EP 2889286A1 and International patent application WO 2015/101501 (PCT/EP2014/078296) entitled Pharmaceutically active compound for use as anti-inflammatory agent.

\section{Appendix A. Supporting information}

Supplementary data associated with this article can be found in the online version at http://dx.doi.org/10.1016/j.ejphar.2016.04.019. 


\section{References}

Alino, S.F., Garcia, D., Uvnäs-Moberg, K., 1986. Effect of intragastric pH, prostaglandins and prostaglandin synthesis inhibitors on the release of gastrin and somatostatin into the gastric lumen of anaesthetized rats. Acta Physiol. Scand. 126 (1), 1-8.

Appleyard, C.B., Alvarez, A., Percy, W.H., 2002. Temporal changes in colonic vascular architecture and inflammatory mediator levels in animal models of colitis. Dig. Dis. Sci. 47, 2007-2014.

Baigent, C., Blackwell, L., Collins, R., Collins, R., Emberson, J., Godwin, J., Peto, R., Buring, J., Hennekens, C., 2009. Aspirin in the primary and secondary prevention of vascular disease: collaborative meta-analysis of individual participant data from randomised trials. Lancet 373 (9678), 1849-1860.

Beckman, J.S., Parks, D.A., Pearson, J.D., Marshall, P.A., Freeman, B.A., 1989. A sensitive fluorometric assay for measuring xanthine dehydrogenase and oxidase in tissues. Free Radic. Biol. Med. 6, 607-615.

Boeer, K., Reinhöfer, M., Lösche, W., 2010. Validation of a procedure to assess ASAresponse in patients with decreased, initial TRAP induced aggregation. Platelets 21 (5), 314-319.

De Cuyper, M., Joniau, M., 1980. Behavior of beef-heart cytochrome c oxidase in reconstituted proteovesicles. A systemtic evaluation of the influence of phospholipid polar headgroup and fatty-acyl side chains. Eur. J. Biochem. 104 (2), 397-405.

Delaney, J.A., Opatrny, L., Brophy, J.M., Suissa, S., 2007. Drug-drug interactions between antithrombotic medications and the risk of gastrointestinal bleeding. Can. Med. Assoc. J. 177 (4), 347-351.

Desmarais, W.T., Bienvenue, D.L., Bzymek, K.P., Holz, R.C., Petsko, G.A., Ringe, D. 2002. The 1.20 A resolution crystal structure of the aminopeptidase from Aeromonas proteolytica complexed with tris: a tale of buffer inhibition. Structure 10 (8), 1063-1072.

Fiorucci, S., Antonelli, E., Migliorati, G., Santucci, L., Morelli, O., Federici, B., Morelli, A., 1998. TNF-alpha processing enzyme inhibitors prevent aspirin-induced TNFalpha release and protect against gastric mucosal injury in rats. Aliment Pharmacol. Ther. 12 (11), 1139-1153.

Foitzik, T., Kruschewski, M., Kroesen, A.J., Hotz, H.G., Eibl, G., Buhr, H.J., 1999. Does glutamine reduce bacterial translocation? A study in two animal models with impaired gut barrier. Int. J. Colorectal Dis. 14, 143-149.

Garrido, C., Galluzzi, L., Brunet, M., Puig, P.E., Didelot, C., Kroemer, G., 2006. Mechanisms of cytochrome c release from mitochondria. Cell Death Differ. 13 (9) 1423-1433.

Ghalanbor, Z., Ghaemi, N., Marashi, S.A., Amanlou, M., Habibi-Rezaei, M., Khajeh, K. Ranjbar, B., 2008. Binding of tris to bacillus licheniformis alpha-amylase can affect its starch hydrolysis activity. Protein Pept. Lett. 15 (2), 212-214.

Gomori, G., 1955. Preparation of Buffers for Use in Enzyme Studies. Methods enzymology, in: Fasman, G.D., (Ed), Handbook of Biochemistry and Molecular Biology, Clevaland, pp.138-146.

Hochain, P., Capet, C., Colin, R., 2000. Digestive complications of aspirin. Rev. Med. interne 21 (1), 50s-59s.

Hoste, E.A., Colpaert, K., Vanholder, R.C., Lameire, N.H., De Waele, J.J., Blot, S.I. Colardyn, F.A., 2005. Sodium bicarbonate versus THAM in ICU patients with mild metabolic acidosis. J. Nephrol. 18 (3), 303-307.

Kallet, R.H., Jasmer, R.M., Luce, J.M., Lin, L.H., Marks, J.D., 2000. The treatment of acidosis in acute lung injury with tris-hydroxymethyl aminomethane (THAM). Am. J. Respir. Crit. Care Med. 161 (4), 1149-1153.

Kovács, T., Varga, G., Erces, D., Tókés, T., Tiszlavicz, L., Ghyczy, M., Boros, M., Kaszaki, J., 2012. Dietary phosphatidylcholine supplementation attenuates inflammatory mucosal damage in a rat model of experimental colitis. Shock 38 (2), 177-185.

Kuebler, W.M., Abels, C., Schuerer, L., Goetz, A.E., 1996. Measurement of neutrophil content in brain and lung tissue by a modified myeloperoxidase assay. Int. J. Microcirc. Clin. Exp. 16 (2), 89-97.

Meade, T.W., Roderick, P.J., Brennan, P.J., Wilkes, H.C., Kelleher, C.C., 1992. Extracranial bleeding and other symptoms due to low dose aspirin and low intensity oral anticoagulation. Thromb. Haemost. 68 (1), 1-6.

Moshage, H., Kok, B., Huizenga, J.R., Jansen, P.L., 1995. Nitrite and nitrate determinations in plasma: a critical evaluation. Clin. Chem. 41, 892-896.

Mroszczak, E.J., Lee, F.W., Combs, D., Sarnquist, F.H., Huang, B.L., Wu, A.T., Tokes, L. G. Maddox, M.L., Cho, D.K., 1987. Ketorolac tromethamine absorption, distribution, metabolism, excretion, and pharmacokinetics in animals and humans. Drug. Metab. Dispos. 15 (5), 618-626.

Nulton-Persson, A.C., Szweda, L.I., Sadek, H.A., 2004. Inhibition of cardiac mitochondrial respiration by salicylic acid and acetylsalicylate. J. Cardiovasc. Pharmacol. 44 (5), 591-595.

Pizzuto, G., Surgo, D., Clementi, M., Marsico, R., Genco, A., Materia, A., Basso, N. 1997. Differential effect of stress on gastric somatostatin, prostaglandin E and gastrin release in the rat. Ital. J. Gastroenterol. Hepatol. 29 (2), 143-147.

Placer, Z.A., Cushman, L.L., Johnson, B.C., 1966. Estimation of product of lipid peroxidation (malonyl dialdehyde) in biochemical systems. Anal. Biochem. 16 (2), 359-364.

Raza, H., John, A., 2012. Implications of altered glutathione metabolism in aspirininduced oxidative stress and mitochondrial dysfunction in HepG2 Cells. PLoS One 7 (4), e36325.

Redlak, M.J., Power, J.J., Miller, T.A., 2005. Role of mitochondria in aspirin-induced apoptosis in human gastric epithelial cells. Am. J. Physiol. Gastrointest. Liver Physiol. 289 (4), G731-G738.

Schrör, K., 1997. Aspirin and platelets: the antiplatelet action of aspirin and its role in thrombosis treatment and prophylaxis. Semin. Thromb. Hemost. 23 (4), 349-356.

Slater, T.F., 1984. Overview of methods used for detecting lipid peroxidation. Methods Enzymol. 105, 283-293.

Sørensen, H.T., Mellemkjaer, L., Blot, W.J., Nielsen, G.L., Steffensen, F.H., McLaughlin, J.K., Olsen, J.H., 2000. Risk of upper gastrointestinal bleeding associated with use of low-dose aspirin. Am. J. Gastroenterol. 95 (9), 2218-2224.

Szarka, A., Horemans, N., Banhegyi, G., Asard, H., 2004. Facilitated glucose and dehydroascorbate transport in plant mitochondria. Arch. Biochem. Biophys. 428 (1), 73-80.

Wallace, J.L., Keenan, C.M., Granger, D.N., 1990. Gastric ulceration induced by nonsteroidal anti-infammatory drugs is a neutrophil-dependent process. Am. J. Physiol. 259, G462-G467.

Watson, W.H., Song, Z., Kirpich, I.A., Deaciuc, I.V., Chen, T., McClain, C.J., 2011. Ethanol exposure modulates hepatic S-adenosylmethionine and S-adenosylhomocysteine levels in the isolated perfused rat liver through changes in the redox state of the NADH/NAD(+) system. Biochim. Biophys. Acta 1812 (5), 613-618. 